\title{
Coadministration of doxorubicin and etoposide loaded in camel milk phospholipids liposomes showed increased antitumor activity in a murine model
}

\author{
This article was published in the following Dove Press journal: \\ International Journal of Nanomedicine \\ 13 April 2015 \\ Number of times this article has been viewed
}

\author{
Hamzah M Maswadeh' \\ Ahmed N Aljarbou' \\ Mohammed S Alorainy ${ }^{2}$ \\ Arshad H Rahmani ${ }^{3}$ \\ Masood A Khan ${ }^{3}$ \\ 'Department of Pharmaceutics, \\ College of Pharmacy, ${ }^{2}$ Department \\ of Pharmacology and Therapeutics, \\ College of Medicine, ${ }^{3}$ College \\ of Applied Medical Sciences, Qassim \\ University, Buraydah, Kingdom \\ of Saudi Arabia
}

\begin{abstract}
Small unilamellar vesicles from camel milk phospholipids (CML) mixture or from 1,2 dipalmitoyl-sn-glycero-3-phosphatidylcholine (DPPC) were prepared, and anticancer drugs doxorubicin (Dox) or etoposide (ETP) were loaded. Liposomal formulations were used against fibrosarcoma in a murine model. Results showed a very high percentage of Dox encapsulation $(\sim 98 \%)$ in liposomes (Lip) prepared from CML-Lip or DPPC-Lip, whereas the percentage of encapsulations of ETP was on the lower side, 22\% of CML-Lip and 18\% for DPPC-Lip. Differential scanning calorimetry curves show that Dox enhances the lamellar formation in CML-Lip, whereas ETP enhances the nonlamellar formation. Differential scanning calorimetry curves also showed that the presence of Dox and ETP together into DPPC-Lip produced the interdigitation effect. The in vivo anticancer activity of liposomal formulations of Dox or ETP or a combination of both was assessed against benzopyrene (BAP)-induced fibrosarcoma in a murine model. Tumor-bearing mice treated with a combination of Dox and ETP loaded into CML-Lip showed increased survival and reduced tumor growth compared to other groups, including the combination of Dox and ETP in DPPC-Lip. Fibrosarcoma-bearing mice treated with a combination of free (Dox + ETP) showed much higher tumor growth compared to those groups treated with CML-Lip-(Dox + ETP) or DPPC-Lip-(Dox + ETP). Immunohistochemical study was also performed to show the expression of tumor-suppressor PTEN, and it was found that the tumor tissues from the group of mice treated with a combination of free (Dox + ETP) showed greater loss of cytoplasmic PTEN than tumor tissues obtained from the groups of mice treated with CML-Lip-(Dox + ETP) or DPPC-Lip-(Dox + ETP).
\end{abstract}

Keywords: combination chemotherapy, BAP-induced tumors, immunohistochemistry

\section{Introduction}

Cancer treatment may include chemotherapy, radiation, and/or surgery. Besides cancerous cells, chemotherapy also targets the normal cells and causes severe side effects such as fatigue, sores in the mouth, diarrhea, nausea, vomiting, constipation, blood disorders, hair loss, and immunosuppression. ${ }^{1,2}$ The existing treatment approaches are not sufficient to cure cancer patients and, therefore, there are continuing efforts to explore novel anticancer strategies. Some clinical regimens comprise multiple noncross-resistant anticancer agents. ${ }^{3,4}$

DNA-modifying agents, such as anthracycline-based topoisomerase II, inhibitors and platinum-based drugs, have been explored in combination chemotherapy against ovarian cancer, advanced breast cancer, endometrial carcinoma, and human lung cancer cell lines or in vivo murine models. ${ }^{4-9}$ There has been drug synergism, where 
the coadministration of two drugs leads to a significantly greater activity than the simple addition of the effects of each drug component. ${ }^{10-13}$

The side effects accompanying anticancer drug combination chemotherapy can potentially be reduced by encapsulating them in the delivery systems. ${ }^{14,15}$ The most effective therapeutic effect can only be achieved by exposing the tumor cells to maximum drug concentrations. For this purpose, it is important to retain two key attributes of liposomal anticancer drug formulations, including 1) extended liposomes (Lip) circulation for passive accumulation in tumors and 2) retention of the encapsulated drug until its delivery to the tumor site. ${ }^{16-19}$

Earlier studies culminated with the observation that coating of Lip with polyethylene glycol (PEG), a synthetic hydrophilic polymer, would improve their stability and lengthen their half-lives in circulation. ${ }^{20-23}$ These PEGcoated Lip are also referred to as sterically stabilized or stealth Lip. A longer blood residence time will result in repeated passages through the tumor microvascular bed of high concentrations of vesicles and, consequently, in a greater efficiency of extravasation per unit volume of convective transport. However, previous studies found that adding PEG to vesicles did not enhance their extravasation into the peritoneal cavity and suggest that PEG may inhibit extravasation. ${ }^{24,25}$ Lipid vesicles with a mean diameter of $100 \mathrm{~nm}$ exhibit longer circulation times than smaller or larger vesicles with the same composition, which can be expected to enhance their extravasation into the peritoneal cavity, leading to a higher liposome accumulation into the lymphatic drainage in tumors. ${ }^{26}$

Camel milk contains fat, protein, lactose, ash, and lactic acid. ${ }^{27}$ The characterization of the phospholipids isolated from camel milk predominantly showed the presence of phosphatidylcoline, phosphatidylethanolamine (PE), phosphatidylinositol, and lysophosphatidylcholine. ${ }^{28}$ Recently we showed that etoposide (ETP) encapsulated into camel milk phospholipids (CML)-Lip possesses greater activity against fibrosarcoma in a mouse model. ${ }^{28}$ Cisplatin showed increased antitumor efficacy in PE-containing Lip. ${ }^{29}$ ETP encapsulated in immunomodulator tuftsin-bearing Lip showed increased antitumor activity. ${ }^{30}$

The present study was aimed to use anticancer drug combination chemotherapy (coadministration of doxorubicin [Dox] and ETP) in Lip prepared from CML. Small unilamellar vesicles $(\sim 100 \mathrm{~nm})$ were prepared from CML mixture or from 1,2 dipalmitoyl-sn-glycero-3phosphatidylcholine (DPPC) loaded with Dox and ETP.
The in vivo activity of various Dox and ETP formulations alone or in combination was tested against experimental fibrosarcoma in a murine model. The Pten, a tumor suppressor gene, is mutated in a wide range of malignancies and cancers. ${ }^{31}$ The PTEN opposes the PI3K-AKT-MDM2 signaling pathway and thus regulates the p53 stability in a phosphatase-dependent manner. ${ }^{32}$ The expression profile of PTEN protein was assessed in treated and untreated groups to evaluate the impact of pten genes in the development and progression of tumors in the treated or untreated groups of mice.

\section{Materials and methods}

DPPC was obtained from Avanti Polar Lipids Inc (Alabaster, AL, USA). Dox and ETP were obtained from Tocris Bioscience (Bristol, UK). Chloroform and methanol used in the study were of spectroscopic grade. Ammonium sulfate, N-2Hydroxyethylpiperzine-N-2-ethanesulfonic acid (HEPES), and Sephadex G-75 were obtained from Thermo Fisher Scientific (Waltham, MA, USA). BAP and PTEN antibody were purchased from Santa Cruz Biotechnology (Dallas, TX, USA). Camel milk was collected from a 6-year-old, red camel 2 months postdelivery, in lactation period, from an Aljarbou farm in Qassim, Saudi Arabia.

\section{Liposome preparation and drug encapsulation}

CML were extracted from camel milk as described earlier. ${ }^{28}$ Lip from DPPC or CML mixture containing Dox or ETP were prepared. The $\mathrm{pH}$-gradient method for Dox encapsulation was used. Briefly, Lip were prepared by dissolving the lipids in chloroform. The solvent was subsequently slowly evaporated using a rotary evaporator. Multilamelar large vesicles (MLVs) were formed by adding $250 \mathrm{mM}$ ammonium sulfate $(\mathrm{pH}=5.3)$. The preparation was then treated by freeze-thaw for ten cycles. The preparation was subsequently extruded ten times each through $200 \mathrm{~nm}$ membrane and $100 \mathrm{~nm}$ polycarbonate membranes using an extruder device Lipex Biomembranes Inc, heated at $50^{\circ} \mathrm{C}$. The size distribution of the DPPC-Lip or CML-Lip before and after extrusion was determined using the Mastersizer by Malvern Instruments Ltd. Subsequently, the extruded Lip were passed through Sephadex G-75, preequilibrated with $25 \mathrm{mM}$ HEPES + $150 \mathrm{mM}$ sodium chloride $(\mathrm{NaCl})(\mathrm{pH}=7.5)$ to create a transmembrane $\mathrm{pH}$ gradient. Lip were incubated with Dox at $60^{\circ} \mathrm{C}$ for 5 minutes. The mixed DPPC/Dox vesicles or Lip prepared from CML (CML-Lip) with encapsulated Dox were separated from unentrapped by filtration through a Sephadex 
G-75 column. Dox was assayed by the automatic high performance liquid chromatography (HPLC) system equipped with ultraviolet (UV)/visible spectroscopic detector 2489 (Waters Corporation, Milford, MA, USA) at $210 \mathrm{~nm}^{28}$

For ETP encapsulation, the thin film hydration method was used. Briefly, Lip from DPPC or CML mixture were prepared by dissolving the lipid and ETP in a mixture of chloroform/methanol. The solvents were subsequently evaporated in a rotary evaporator. MLVs were formed by adding normal saline. The preparation was then treated by freeze-thaw and extruded as described above. The extruded DPPC-Lip-ETP or CML-Lip-ETP were separated from free ETP through a Sephadex G-75 column. ETP was assayed by the automatic HPLC system equipped with UV/visible spectroscopic detector at $210 \mathrm{~nm}$.

\section{Drug release experiments}

The release of Dox and ETP from Lip at $37^{\circ} \mathrm{C}$ was assayed as follows: Dox containing Lip were first passed through a Sephadex G-75 column equilibrated with 25 mM HEPES + $150 \mathrm{mM} \mathrm{NaCl}$ ( $\mathrm{pH}$ 7.5), whereas ETP-containing Lip were passed through a Sephadex G-75 column equilibrated with normal saline to remove the free drug. The samples were then placed in a plastic tube and incubated in a water bath maintained at $37^{\circ} \mathrm{C}$. Aliquots were removed at various times, and the released drug was separated employing Sephadex G-75 column. Vesicles were disrupted with ethanol and the entrapped drugs were assayed by HPLC/UV spectroscopy at $210 \mathrm{~nm}$.

\section{Differential scanning calorimetry}

A conventional differential scanning calorimetry (DSC) technique was applied for the study of DPPC MLVs and CML MLVs using a Shimatzu DSC-60 calorimeter. DPPC or CML mixture with Dox and ETP separately and in combination were dissolved in chloroform or chloroform/methanol. The solvents were then evaporated by rotary evaporator under vacuum at temperatures above the transition temperature of the phospholipids. For measurements, the dry residue was dispersed in appropriate amounts of double distilled water by vortexing. An aliquot (approximately $5 \mathrm{mg}$ ) was sealed into stainless steel capsules obtained from Shimadzu. DSC curves were obtained on a Shimadzu DSC-60 calorimeter. Prior to scanning, the samples were held above their phase transition temperature for 1-2 minutes to ensure equilibration. All samples were scanned at least twice until identical curves were obtained using a scanning rate of $3^{\circ} \mathrm{C} /$ minute for DPPC and $10^{\circ} \mathrm{C} /$ minute for CML mixture.

\section{BAP-induced tumor model}

Female BALB/C mice (20-25 grams of weight) were obtained from the animal house facility of King Saud University, Riyadh, Saudi Arabia. All experiments were approved by the animal ethics committee of the College of Pharmacy, Qassim University, Buraydah, Saudi Arabia. Tumors were induced chemically by injecting BAP dissolved in sesame oil at a dose of $250 \mu \mathrm{g} /$ mouse through subcutaneous route as described earlier. ${ }^{30}$ After $100-120$ days post-BAP injections mice developed palpable tumors.

\section{Treatment of tumor-bearing mice}

Tumor-bearing mice were divided into 12 different treatment groups, and each group comprised of ten mice. Mice were divided into the following groups:

1) untreated control; 2) sham DPPC-Lip; 3) sham CMLLip; 4) free Dox $5 \mathrm{mg} / \mathrm{kg}$; 5) Free ETP $5 \mathrm{mg} / \mathrm{kg}$; 6) free Dox + ETP, 2.5+2.5 mg/kg; 7) DPPC-Lip-Dox, $5 \mathrm{mg} / \mathrm{kg}$; 8) DPPC-Lip-ETP, $5 \mathrm{mg} / \mathrm{kg}$; 9) DPPC-Lip-(Dox + ETP), $2.5+2.5 \mathrm{mg} / \mathrm{kg}$; 10) CML-Lip-Dox, $5 \mathrm{mg} / \mathrm{kg}$; 11) CML-LipETP, $5 \mathrm{mg} / \mathrm{kg}$; 12) CML-Lip-(Dox + ETP) and DPPC-Lip(Dox + ETP), $2.5+2.5 \mathrm{mg} / \mathrm{kg}$.

Tumors were measured using Vernier calipers until they reached a volume of $150-200 \mathrm{~mm}^{3}$. Various formulations of anticancer drugs Dox and ETP alone or in combination were administered intraperitoneally to treat tumor-bearing mice once a week for 3 weeks. The first day of treatment was considered day 0 . The size of the tumors were calculated according to following formula:

$$
\mathrm{V}=\mathrm{D} \times \mathrm{d} 2 \times \pi / 6
$$

where $\mathrm{V}$ is tumor volume, $\mathrm{D}$ is biggest dimension, $\mathrm{d}$ is smallest dimension. The efficacy of the treatment was assessed by measuring the change in tumor size and analyzing the survival of the untreated or treated mice.

\section{Immunohistochemical study}

After 3 weeks of chemotherapy, two mice from each group were sacrificed, and paraffin embedded tissue-blocks were made for immunohistochemical staining. The expression of PTEN protein was evaluated by immunohistochemistry as described earlier. ${ }^{33}$ Briefly formalin-fixed, paraffin-embedded tissues were cut in 5-10 $\mu \mathrm{m}$ thin sections followed by deparaffinization, rehydration, and rinsing in phosphatebuffered saline (PBS). After antigen retrieval, slides were incubated with a monoclonal antibody for PTEN (Santa Cruz Biotechnology), followed by a biotinylated secondary 
antibody. Sections were washed in PBS and then incubated with streptavadin peroxidase. Finally, diaminobenzidine was used as a chromogen, and sections were counterstained with haematoxylin. A total of four fields from tissue sections were chosen, and 100 cells from each field were calculated at a magnification of $100 \times$. Tumor cases were considered as PTEN positive if more than $5 \%$ of cells showed cytoplasmic positivite.

\section{Statistics}

Survival of the mice was analyzed by log-rank test using the Kaplan-Meier curve. The sizes of tumors were compared by one-way ANOVA using Prism software version 6.0 (GraphPad Software Inc, San Diego, CA, USA).

\section{Results and discussion Dox/ETP encapsulation and drug release}

The vesicle size distribution of Lip after ten times extrusions through $200 \mathrm{~nm}$ followed by ten times extrusion through $100 \mathrm{~nm}$ polycarbonate membrane was $75.5 \mathrm{~nm}$ and $113 \mathrm{~nm}$ for CML-Lip and DPPC-Lip, respectively (results not shown).

Dox was encapsulated into DPPC-Lip or into CML-Lip within 5 minutes at $60^{\circ} \mathrm{C}$ in response to a transmembrane ammonium sulfate gradient, with trapping efficiency more than 98\%. ETP was encapsulated into DPPC-Lip and CML-Lip with trapping efficiency not more than $18 \%$ and $22 \%$, respectively. The extent of Dox and ETP uptake was highly dependent on drug solubility and the method used for drug encapsulation. Dox is an amphiphilic drug that can be encapsulated in the hydrophilic as well as in the lipophilic compartments of Lip. Also, the pH-gradient method can be used for Dox encapsulation, with a very high efficiency. ETP is lipophilic drug that can be incorporated in a small quantity into the bilayer of Lip.

The retention of Dox or ETP within Lip was monitored at $37^{\circ} \mathrm{C}$ in a buffer for Dox and in normal saline for ETP. The release of Dox from DPPC-Lip or CML-Lip after 24 hours was $24 \%$ and $18 \%$, respectively. The release of ETP after 24 hours from DPPC-Lip or CML-Lip was $26 \%$ and 19\%, respectively (Figure 1).

\section{Thermal effects of Dox or ETP in multilamellar vesicles composed of DPPC or CML}

We have applied conventional DSC to study the thermal effects of Dox or ETP in multilamellar vesicles composed of DPPC or a CML. The DSC curves for DPPC with or without drug(s) are shown in Figure 2, and the corresponding

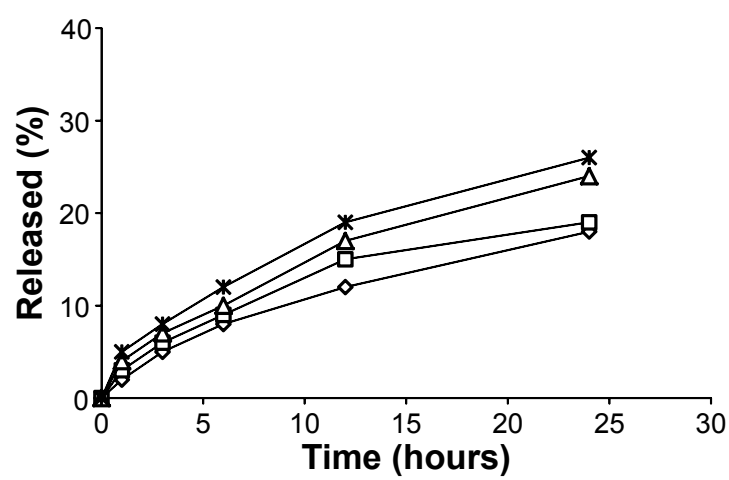

Figure I Percent of drug released from liposomes at $37^{\circ} \mathrm{C}$. Notes: $(\diamond)$ CML-liposomes with doxorubicin; $(\square)$ CML-liposomes with etoposide; $(\Delta)$ DPPC-liposomes with doxorubicin; (ж) DPPC-liposomes with etoposide. Abbreviations: CML, camel milk phospholipids; DPPC, I,2 dipalmitoyl-sn-glycero3-phosphatidylcholine.

calorimetric results are shown in Table 1. The size of a cooperative unit $(\mathrm{CU})$ of the main transition enthalpies was calculated. Table 1 and Figure 2A show that DPPC multilamellar vesicles exist in the gel phase from temperatures lower than $36^{\circ} \mathrm{C}$, and in the liquid crystalline phase for temperature higher than $42^{\circ} \mathrm{C}$. The presence of $3 \%$ Dox in

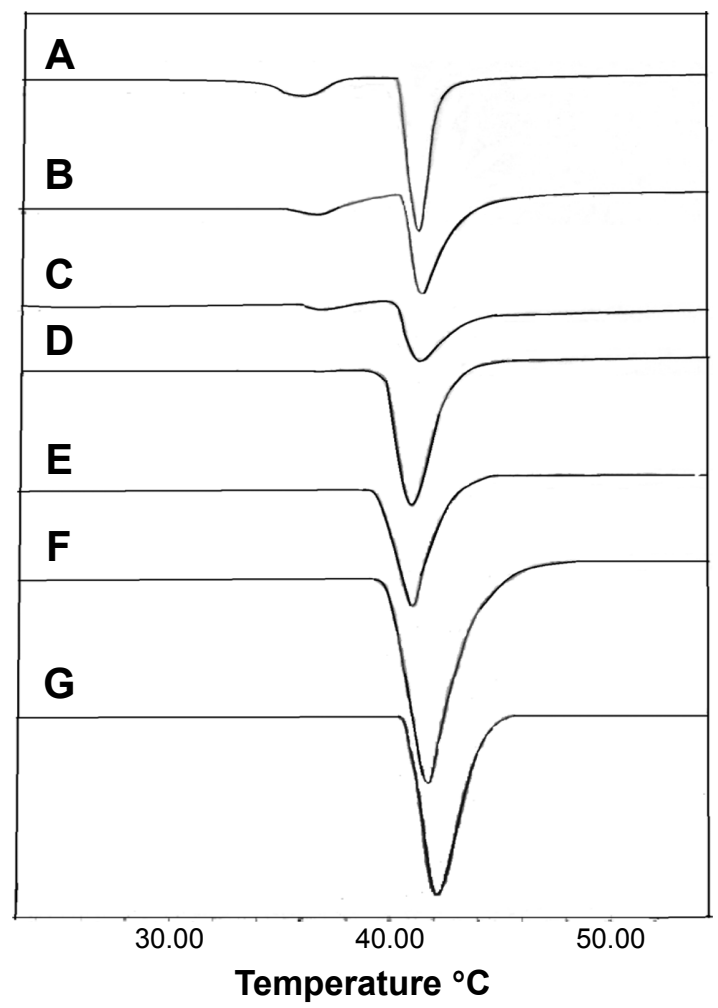

Figure 2 DSC curves.

Notes: DSC curves for (A) DPPC, (B) DPPC with $3 \%(w / w)$ doxorubicin, (C) DPPC with $6 \%(\mathrm{w} / \mathrm{w})$ doxorubicin, (D) DPPC with $3 \%(\mathrm{w} / \mathrm{w})$ etoposide, (E) DPPC with $6 \%(w / w)$ etoposide, (F) DPPC with $3 \%(w / w)$ doxorubicin and $3 \%(w / w)$ etoposide, and $(\mathbf{G})$ DPPC with $6 \%(w / w)$ doxorubicin and $6 \%(w / w)$ etoposide.

Abbreviations: DPPC, I,2 dipalmitoyl-sn-glycero-3-phosphatidylcholine; DSC, differential scanning calorimetry. 
Table I Data from DSC curves for different mixtures of DPPC with doxorubicin and etoposide either alone or in combination

\begin{tabular}{|c|c|c|c|c|c|c|c|c|}
\hline \multirow[t]{2}{*}{ Composition } & \multicolumn{2}{|c|}{ Pretransition $\left({ }^{\circ} \mathrm{C}\right)$} & \multicolumn{6}{|c|}{ Main lipid phase transition $\left({ }^{\circ} \mathrm{C}\right)$} \\
\hline & $\begin{array}{l}\Delta H_{\text {pre }} \\
(\mathrm{Kcal} / \mathrm{mol})\end{array}$ & $\begin{array}{l}\text { Peak } \\
\left({ }^{\circ} \mathrm{C}\right)\end{array}$ & $\begin{array}{l}\text { Start } \\
\left({ }^{\circ} \mathrm{C}\right)\end{array}$ & $\begin{array}{l}\text { Peak } \\
\left({ }^{\circ} \mathrm{C}\right)\end{array}$ & $\begin{array}{l}\text { End } \\
\left({ }^{\circ} \mathrm{C}\right)\end{array}$ & $\begin{array}{l}\Delta H \\
(\text { Kcal/mol) }\end{array}$ & $\begin{array}{l}\Delta T_{1 / 2} \\
\left({ }^{\circ} \mathrm{C}\right)\end{array}$ & $\begin{array}{l}\text { Cooperative } \\
\text { unit (CU) }\end{array}$ \\
\hline DPPC & 0.59 & 36.67 & 40.76 & 41.67 & 45.29 & 5.64 & 1.25 & 97 \\
\hline DPPC + doxorubicin $(97: 3 \mathrm{w} / \mathrm{w})$ & 0.35 & 35.86 & 40.50 & 42.01 & 46.40 & 4.72 & 1.75 & 83 \\
\hline DPPC + doxorubicin $(94: 6 \mathrm{w} / \mathrm{w})$ & 0.27 & 35.80 & 40.70 & 42.16 & 48.45 & 3.63 & 2.56 & 74 \\
\hline DPPC + etoposide $(97: 3 \mathrm{w} / \mathrm{w})$ & - & - & 40.16 & 41.42 & 44.84 & 5.50 & 1.55 & 80 \\
\hline DPPC + etoposide $(94: 6 \mathrm{w} / \mathrm{w})$ & - & - & 39.82 & 41.40 & 47.43 & 5.30 & I.7I & 75 \\
\hline DPPC + doxrubicin + etoposide $(94: 3: 3 \mathrm{w} / \mathrm{w})$ & - & - & 39.81 & 41.87 & 44.79 & 12.38 & 2.43 & 23 \\
\hline DPPC + doxrubicin + etoposide $(88: 6: 6 \mathrm{w} / \mathrm{w})$ & - & - & 39.93 & 42.0 & 46.55 & 8.2 & 2.50 & 33 \\
\hline
\end{tabular}

Abbreviations: $\Delta H$, enthalpy of fusion; $\Delta T_{1 / 2}$, half-width for the main transition peak; CU, cooperative unit; DPPC, I,2 dipalmitoyl-sn-glycero-3-phosphatidylcholine; DSC, differential scanning calorimetry; pre, pretransition.

DPPC multilamellar vesicles (Figure 2B) causes a decrease in the phase transition temperature from $36.6^{\circ} \mathrm{C}$ to $35.8^{\circ} \mathrm{C}$ and decrease in the enthalpy of fusion $(\Delta H)$ of pretransition and main transition peaks with increase in main peak transition from $41.6^{\circ} \mathrm{C}$ to $42^{\circ} \mathrm{C}$ and an increase of the width of the peak for the main lipid phase transition from $1.25^{\circ} \mathrm{C}$ to $1.75^{\circ} \mathrm{C}$. Also, a decrease in the CU from 97 to 84 was observed. By increasing the concentration of Dox to 6\% (Figure 2C) the width of the peak of the main lipid phase transition increases to 2.56 and the $\mathrm{CU}$ decreases to 74 . The area of the phase transition $\Delta H$ and the height of pretransition and main transition peaks were decreased as the concentration of Dox increased. At higher concentrations of Dox an abolishment of the pretransition peak was observed (data not shown).

The pretransition peak was completely abolished in the presence of $3 \%$ or $6 \%$ ETP (Figure $2 \mathrm{D}$ and E). The $\Delta H$ of the main transition peak was decreased, and the width of the peak was increased. Also, the size of CU was decreased.

In this study, the presence of Dox and ETP (3\% or 6\%) together in the DPPC multilamellar vesicles produces an increase in $\Delta H$, height, and width of the main transition peak, with abolishment of the pretransition peak (Figure $2 \mathrm{~F}$ and G; Table 1). Also, the size of the CU was dramatically reduced. It can be concluded that the presence of Dox and ETP together in the DPPC multilamellar vesicles produces the interdigitation effect. The increase of $\Delta H$ is the most diagnostic parameter for showing the interdigitation effect; also, the increases in the height and width of the main transition peak with a decrease in the $\mathrm{CU}$ are strong indicators of interdigitation effect. ${ }^{32,33}$ This interdigitation effect was not observed for Dox or ETP alone.

Figure 3A shows the DSC curve for camel phospholipids mixture. The DSC curve shows that the first highest transition peak of this mixture was observed at $42^{\circ} \mathrm{C}$. It represents the mixture of phosphatidylcoline, PE, phosphatidylinositol, and lysophosphatidylcholine phospholipids mainly present in camel milk. In our recent study, the identification of these phospholipids was done by gas chromatography-mass spectrometry and HPLC. ${ }^{28}$ Due to the presence of PE in CML mixture, a second and third peak were also observed at $118^{\circ} \mathrm{C}$ and $180^{\circ} \mathrm{C}$, respectively. It is well known that natural $\mathrm{PE}$ is the most abundant lipid in cellular membranes and can form either lamellar or nonlamellar phases. The second broad curve started at $85^{\circ} \mathrm{C}$ and ended at $122^{\circ} \mathrm{C}$, indicating that the liquid crystalline phase and the hexagonal phase (fully hydrated) of PE phospholipids coexist. Transition from liquid-crystalline phase of nonlamellar hexagonal phase transition temperature $\left(\mathrm{T}_{\mathrm{h}}\right)$ has a low entropy $(\Delta H)$ (Table 2$)$. The third peak at $188^{\circ} \mathrm{C}$ represents the nonlamellar cubic phase (cubic formation) of camel milk PE phospholipids.

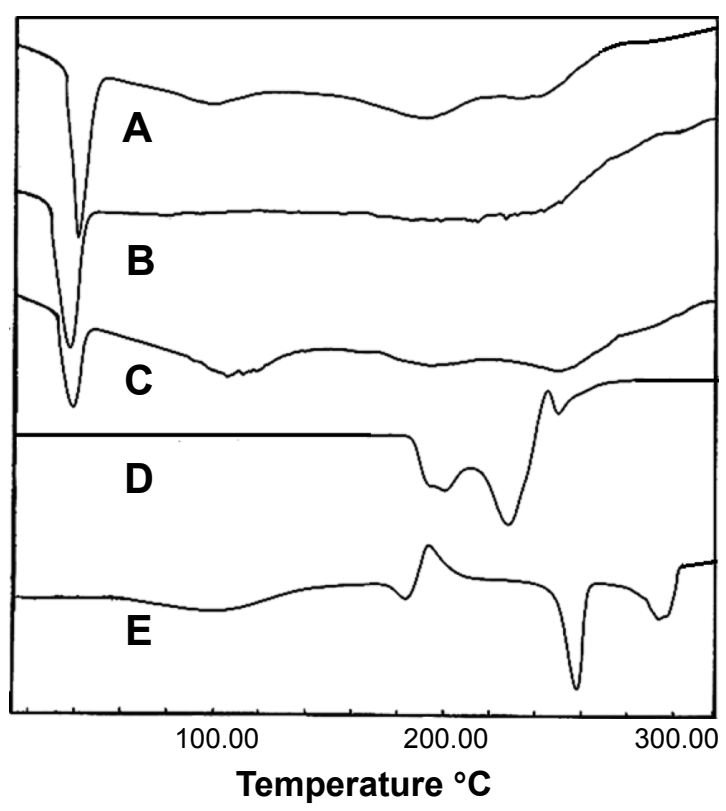

Figure 3 DSC curves.

Notes: DSC curves for (A) camel milk phospholipid mixture, (B) camel milk phospholipid mixture with doxorubicin $94: 6 \mathrm{w} / \mathrm{w}$, (C) camel milk phospholipid mixture with etoposide 94:6 w/w, (D) pure doxorubicin, and (E) pure etoposide. Abbreviation: DSC, differential scanning calorimetry. 
Table 2 Data from DSC curves for camel phospholipid mixture alone or with doxorubicine/etoposide

\begin{tabular}{lllllll}
\hline Composition & $\mathbf{T}_{\mathrm{m}}\left({ }^{\circ} \mathbf{C}\right)$ & $\Delta \boldsymbol{H}_{\mathrm{m}}(\mathbf{j} / \mathbf{g})$ & $\mathbf{T}_{\mathrm{h}}$ & $\Delta \boldsymbol{H}_{\mathrm{h}}(\mathbf{j} / \mathbf{g})$ & $\boldsymbol{T}_{\text {Cubic }}\left({ }^{\circ} \mathbf{C}\right)$ & $\Delta \boldsymbol{H}_{\text {Cubic }}(\mathbf{j} / \mathbf{g})$ \\
\hline Camel phospholipid mixture & 41.49 & 27.07 & 95.08 & 5.16 & 188.0 & 13.52 \\
Camel phospholipids + doxorubicin $(94: 6 \mathrm{w} / \mathrm{w})$ & 39.99 & 30.47 & Disappear & - & Disappear & - \\
Camel phospholipids + etoposide $(94: 6 \mathrm{w} / \mathrm{w})$ & 39.56 & 14.68 & 105.74 & 10.2 & 194.98 & 5.07 \\
\hline
\end{tabular}

Abbreviations: DSC, differential scanning calorimetry; $T_{m}$, main transition temperature; $\Delta H_{m}$, enthalpy of fusion for hexagonal phase transition; $T_{h}$, hexagonal phase transition temperature; $\Delta H_{h^{\prime}}$, enthalpy of fusion for the hexagonal phase transition; $T_{\text {cubic }}$ cubic phase transition temperature; $\Delta H_{\text {cubic }}$ enthalpy of fusion for the cubic phase transition.

Figure 3D shows the DSC for pure Dox. The addition of $6 \%$ of Dox to the CML enhances the lamellar formation by complete abolishment of both hexagonal and cubic phases (Figure 3B). Small peaks were also observed between $200^{\circ} \mathrm{C}$ and $230^{\circ} \mathrm{C}$ due to Dox incorporation into CML. Also, the presence of Dox reduces the main transition temperature from $41.49^{\circ} \mathrm{C}$ to $39.99^{\circ} \mathrm{C}$, with an increase in the $\Delta H$ (Table 2).

Figure 3E shows the DSC curve for pure ETP. The addition of $6 \%$ of ETP to the CML decreases the main transition temperature to $39.56^{\circ} \mathrm{C}$ and also decreases $\Delta H$ (Table 2). The DSC curve in Figure 3C shows that the presence of ETP into CML mixture increases the hexagonal and cubic phase transition and produces an increase in nonlamellar formation.

\section{Coadministration of Dox and ETP in CML-Lip showed increased activity against experimental fibrosarcoma in a murine model}

The in vivo activity of formulations of Dox or ETP or a combination of both was assessed against fibrosarcoma in a murine model. Chemotherapy was started when tumors attained the size in the range of $150-200 \mathrm{~mm}^{3}$. Fibrosarcomabearing mice were treated with a single weekly dose of $5 \mathrm{mg} / \mathrm{kg}$ of free or liposomal formulations of Dox or ETP or a combination of both for 3 weeks. Among the all treatment groups, the tumors showed the least progression or delayed growth in the group of mice treated with CML-Lip-(Dox + ETP) compared to the groups treated with free (Dox + ETP) or DPPC-Lip-(Dox + ETP) (Figure 4A and B). Once chemotherapy was stopped, there was a sharp progression in the size of tumors, particularly in the groups of mice treated with free formulations of drugs (Figure 4A and B).

Besides assessing the effect of chemotherapy on the progression of the tumor size, the effects of various therapeutic formulations was also assessed on the survival of tumor-bearing mice. Consistent with its effect in restricting the growth of tumors, chemotherapy with CML-Lip(Dox + ETP) imparted greater survival compared to those treated with free (Dox + ETP) or DPPC-Lip-(Dox + ETP) (Figure 5A and B). Tumor-bearing mice treated with CML-Lip-(Dox + ETP) showed $\sim 40 \%$ survival on day 90 postchemotherapy, whereas mice in the groups treated with DPPC-Lip-(Dox + ETP) showed 30\% survival (Figure 5A and B). Mice treated with free Dox or free ETP or a combination of both died within 60 days of starting chemotherapy (Figure 5A and B).

The characterization of the phospholipids isolated from camel milk showed the presence of PE. ${ }^{28}$ Earlier studies have shown the increased antitumor efficacy of PE-containing Lip loaded with cisplatin or ETP. ${ }^{29,30}$ This is also confirmed by

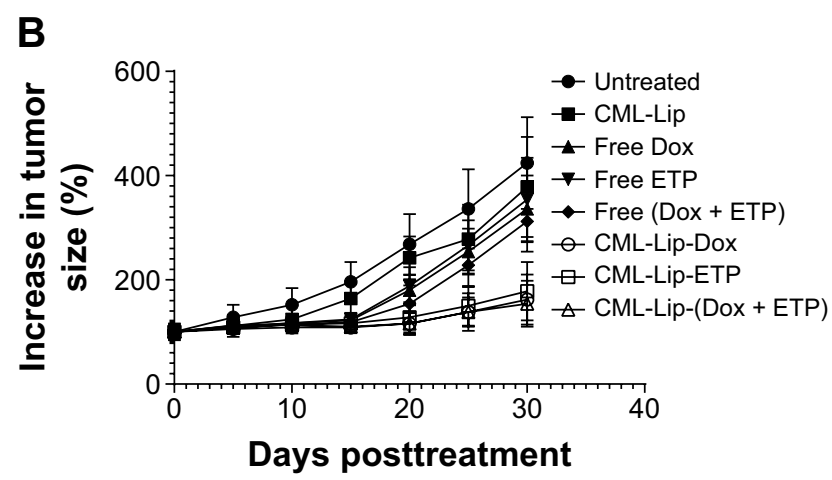

Figure 4 Effects of liposomal formulations of etoposide and doxorubicin against BAP-induced tumors.

Notes: Mice treated with (A) DPPC-Lip-(Dox + ETP), (B) CML-Lip-(Dox + ETP), or showed delayed tumor growth as compared to free drugs (P $<0.00$ I); Data are values \pm $\mathrm{SD}(\mathrm{n}=10$ at initiation of therapy; the number varies at later time points due some mortalities).

Abbreviations: BAP, benzopyrene; CML-Lip, camel milk phospholipids liposomes; Dox, doxorubicin; DPPC-Lip, I,2 dipalmitoyl-sn-glycero-3-phosphatidylcholine liposomes; ETP, etoposide; SD, standard deviation. 

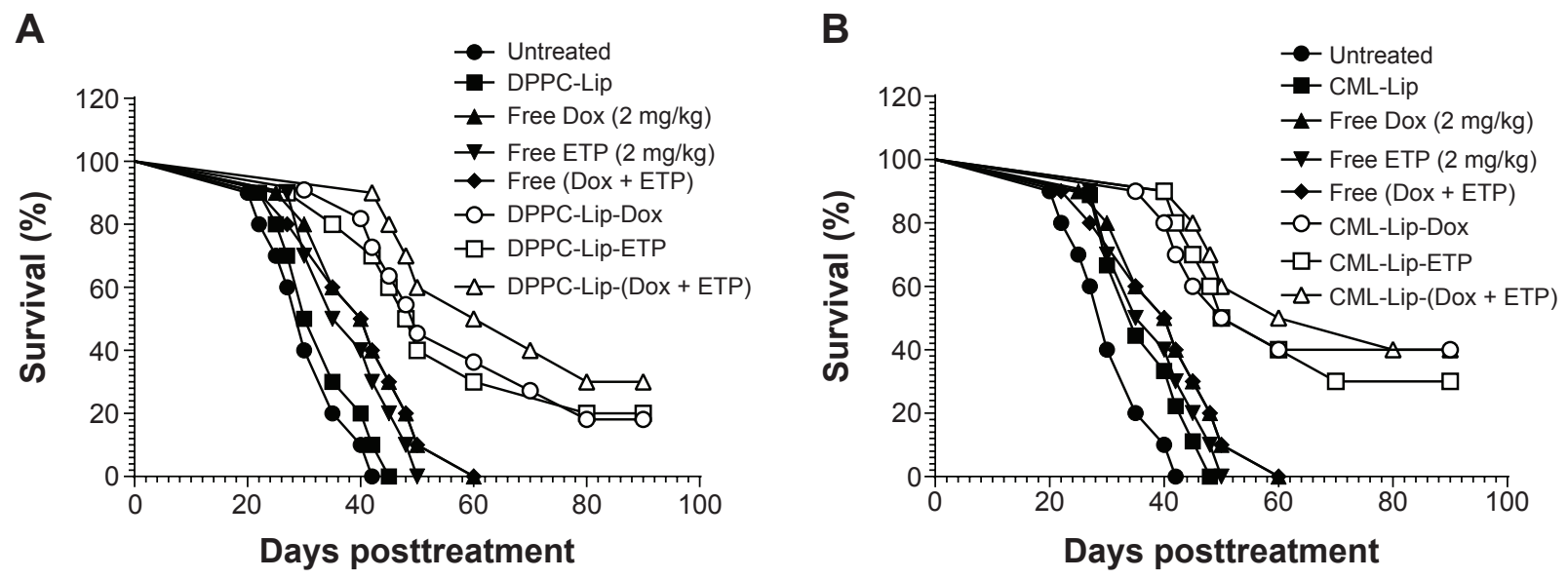

Figure 5 Effects of liposomal formulations of etoposide and doxorubicin on the survival of tumor-bearing mice.

Notes: Various formulations of doxorubicin or etoposide loaded in (A) DPPC and (B) CML-Lip at the dose of $5 \mathrm{mg} / \mathrm{kg}$ were administered in tumor-bearing mice weekly for 3 weeks; The first day of treatment was considered day 0; DPPC-Lip-(Dox + ETP) versus free (Dox + ETP) $(P=0.0021)$; CML-Lip-(Dox + ETP) versus free (Dox + ETP) $(P=0.0025)$; DPPC-Lip-(Dox + ETP) versus DPPC-Lip $(P<0.000 I)$; CML-Lip (Dox + ETP) versus CML-Lip $(P=0.0002)$.

Abbreviations: CML-Lip, camel milk phospholipids liposomes; Dox, doxorubicin; DPPC-Lip, I,2 dipalmitoyl-sn-glycero-3-phosphatidylcholine liposomes; ETP, etoposide.
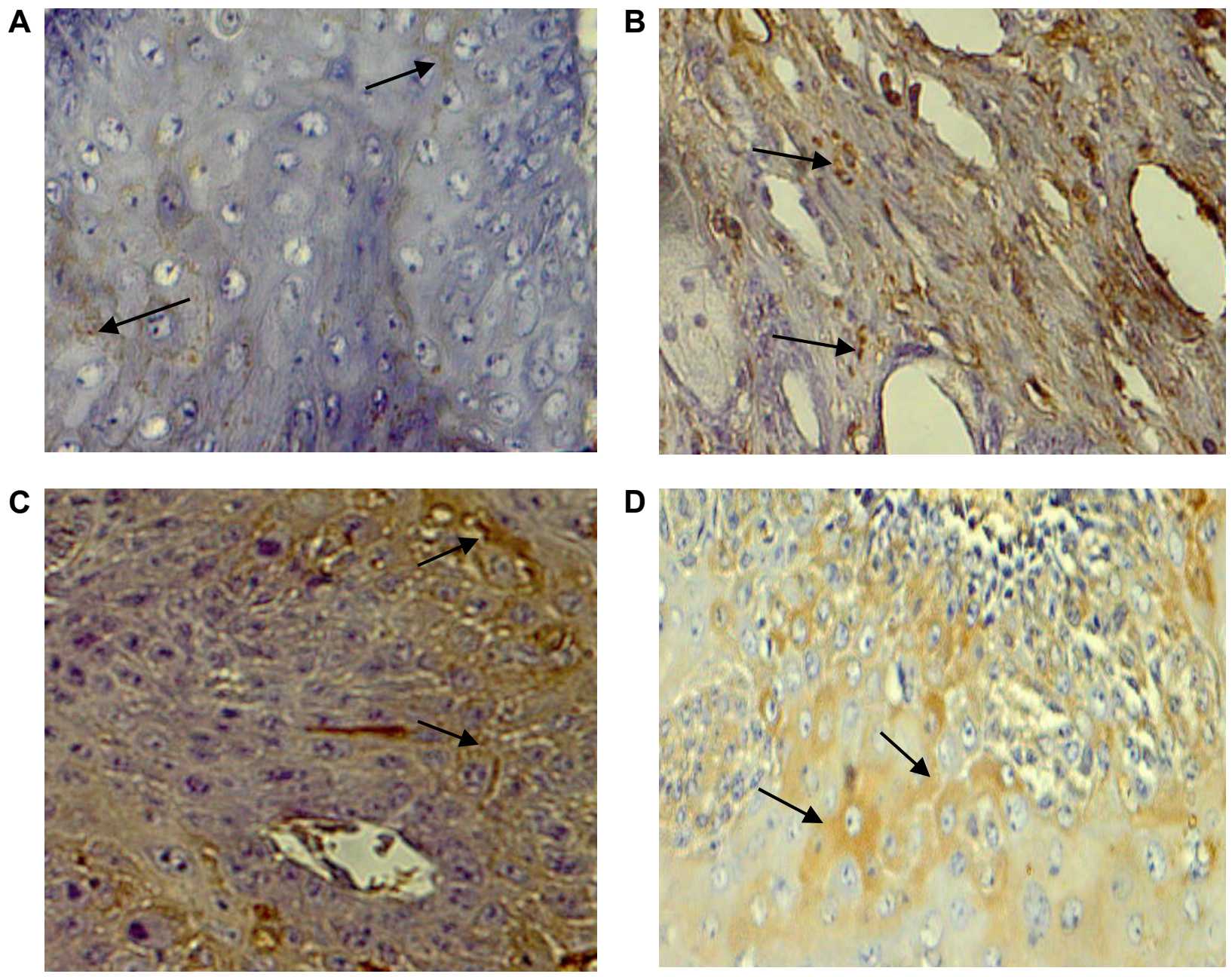

Figure 6 PTEN expression in (A) untreated control, (B) free Dox + free ETP, (C) DPPC-Lip-(Dox + ETP), and (D) CML-Lip-(Dox + ETP)-treated fibrosarcomabearing mice.

Note: The arrows indicate expression of tumor suppressor gene PTEN.

Abbreviations: CML-Lip, camel milk phospholipids liposomes; Dox, doxorubicin; DPPC-Lip, I,2 dipalmitoyl-sn-glycero-3-phosphatidylcholine liposomes; ETP, etoposide. 
the results of the present study that showed greater efficacy of CML-Lip-(Dox + ETP) compared to the DPPC-Lip-(Dox + ETP) or free (Dox + ETP) as CML show a substantial presence of PE. Furthermore, the codelivery of Dox and ETP by DPPC-Lip (DPPC-Lip-[Dox + ETP]) or camel milk Lip (CML-Lip-[Dox + ETP]) was more effective than single drug formulation such as DPPC-Lip-Dox, DPPC-Lip-ETP, CML-Lip-Dox, or CML-Lip-ETP, suggesting the use of combination of drugs to treat cancers.

\section{Loss of tumor suppressor PTEN in BAP-induced tumors in mice}

pten is a tumor suppressor gene, and loss of PTEN protein was noticed according to progression of tumors. ${ }^{31-33}$ Thus, in the present study, the expression of PTEN was studied to show the status of progression of tumors in the groups of mice treated with free Dox + free ETP) or DPPC-Lip-(Dox + ETP) or CML-Lip-(Dox + ETP). Tumor tissues taken from the untreated group showed a significant loss of PTEN (Figure 6A). The groups of mice treated with CML-Lip-(Dox + ETP) or DPPC-Lip-(Dox + ETP) showed less PTEN loss as compared to those treated with free Dox + free ETP or untreated control (Figure 6A-D). The results of the present study reaveled that the loss of PTEN expression is associated with the growth of tumors in the mice. Fibrosarcoma-bearing mice treated with a combination of free (Dox + ETP) showed much higher tumor growth and thus greater PTEN loss, whereas the groups treated with CML-Lip-(Dox + ETP) or DPPC-Lip-(Dox + ETP) showed restricted growth and thus reduced PTEN loss.

\section{Conclusion}

It can be concluded that the codelivery of Dox and ETP loaded in CML-Lip showed superior antitumor activity in a murine model, making CML-Lip-(Dox + ETP) a novel formulation for optimizing the delivery of anticancer drugs in a combination chemotherapy.

\section{Acknowledgment}

This work is part of a project supported by grants from the National Plan for Science, Technology and Innovation (10-NAN-1226-09)/Qassim University, Buraydah, Saudi Arabia.

\section{Disclosure}

The authors report no conflict of interest in this work.

\section{References}

1. Minotti G, Menna P, Salvatorelli E, Cairo G, Gianni L. Anthracyclines: molecular advances and pharmacologic developments in antitumor activity and cardiotoxicity. Pharmacol Rev. 2004;56(2):185-229.

2. Wang D, Lippard SJ. Cellular processing of platinum anticancer drugs. Nat Rev Drug Discov. 2005;4(4):307-320.

3. Jia J, Zhu F, Ma X, Cao Z, Li Y, Chen YZ. Mechanisms of drug combinations: interaction and network perspectives. Nat Rev Drug Discov. 2009;8(2):111-128.

4. Nitiss JL. Targeting DNA topoisomerase II in cancer chemotherapy. Nat Rev Cancer. 2009;9(5):338-350.

5. Kelland L. The resurgence of platinum-based cancer chemotherapy. Nat Rev Cancer. 2007;7(8):573-584.

6. Martoni A, Bellucco A, Canova N, Pannuti F. Four-year analysis of platinum and anthracycline combination for ovarian cancer. Oncology. 1989;46(2):109-116.

7. Nielsen OS, Dombernowsky P, Mouridsen H, et al. Epirubicin is not superior to doxorubicin in the treatment of advanced soft tissue sarcomas. The experience of the EORTC Soft Tissue and Bone Sarcoma Group. Sarcoma. 2000;4(1-2):31-35.

8. Thigpen JT, Brady MF, Homesley HD, et al. Phase III trial of doxorubicin with or without cisplatin in advanced endometrial carcinoma: a gynecologic oncology group study. J Clin Oncol. 2004;22(19):3902-3908.

9. Bigioni M, Benzo A, Irrissuto C, et al. Antitumour effect of combination treatment with Sabarubicin (MEN 10755) and cis-platin (DDP) in human lung tumour xenograft. Cancer Chemother Pharmacol. 2008; 62(4):621-629.

10. Chou TC, Talalay P. Analysis of combined drug effects: a new look at a very old problem. Trends Pharmacol Sci. 1983;4:450-454.

11. Chou TC, Talalay P. Quantitative analysis of dose-effect relationships: the combined effects of multiple drugs or enzyme inhibitors. Adv Enzyme Regul. 1984;22:27-55.

12. Chou TC. Drug combination studies and their synergy quantification using the Chou-Talalay method. Cancer Res. 2010;70(2):440-446.

13. Berenbaum MC. What is synergy? Pharmacol Rev. 1989;41(2): 93-141.

14. Zhao L, Wientjes MG, Au JL. Evaluation of combination chemotherapy: integration of nonlinear regression, curve shift, isobologram, and combination index analyses. Clin Cancer Res. 2004;10(23):7994-8004.

15. Peer D, Karp JM, Hong S, Farokhzad OC, Margalit R, Langer R. Nanocarriers as an emerging platform for cancer therapy. Nat Nanotechnol. 2007;2(12):751-760.

16. Davis ME, Chen ZG, Shin DM. Nanoparticle therapeutics: an emerging treatment modality for cancer. Nat Rev Drug Discov. 2008;7(9): 771-782.

17. Blume G, Cevc G. Liposomes for the sustained drug release in vivo. Biochim Biophys Acta. 1990;1029(1):91-97.

18. Woodle MC, Lasic DD. Sterically stabilized liposomes. Biochim Biophys Acta. 1992;1113(2):171-199.

19. Lasic DD, Martin FJ, Gabizon A, Huang SK, Papahadjopoulos D. Sterically stabilized liposomes: a hypothesis on the molecular origin of the extended circulation times. Biochim Biophys Acta. 1991; 1070(1):187-192.

20. Gabizon AA. Selective tumor localization and improved therapeutic index of anthracyclines encapsulated in long-circulating liposomes. Cancer Res. 1992;52(4):891-896.

21. Gabizon A, Papahadjopoulos D. Liposome formulations with prolonged circulation time in blood and enhanced uptake by tumors. Proc Natl Acad Sci U S A. 1988;85(18):6949-6953.

22. Gabizon A, Shiota R, Papahadjopoulos D. Pharmacokinetics and tissue distribution of doxorubicin encapsulated in stable liposomes with long circulation times. J Natl Cancer Inst. 1989;81(19):1484-1488.

23. Gabizon A, Price DC, Huberty J, Bresalier RS, Papahadjopoulos D. Effect of liposome composition and other factors on the targeting of liposomes to experimental tumors: biodistribution and imaging studies. Cancer Res. 1990;50(19):6371-6378. 
24. Klibanov AL, Maruyama K, Torchilin VP, Huang L. Amphipathic polyethyleneglycols effectively prolong the circulation time of liposomes. FEBS Lett. 1990;268(1):235-237.

25. Senior J, Delgado C, Fisher D, Tilcock C, Gregoriadis G. Influence of surface hydrophilicity of liposomes on their interaction with plasma protein and clearance from the circulation: studies with poly(ethylene glycol)-coated vesicles. Biochim Biophys Acta. 1991;1062(1):77-82.

26. Allen TM, Austin GA, Chonn A, Lin L, Lee KC. Uptake of liposomes by cultured mouse bone marrow macrophages: influence of liposome composition and size. Biochim Biophys Acta. 1991;1061(1):56-64.

27. Konuspayeva G, Faye B, Loiseau G. The composition of camel milk: a meta-analysis of the literature data. J Food Compos Anal. 2008; 22:95-101.

28. Maswadeh HM, Aljarbou AN, Alorainy MS, Alsharidah MS, Khan MA. Etoposide incorporated into camel milk phospholipids liposomes shows increased activity against fibrosarcoma in a mouse model. Bio Med Research Int. 2015:11. DOI: http:// dx.doi.org/10.1155/2015/743051.
29. Hwang TL, Lee WR, Hua SC, Fang JY. Cisplatin encapsulated in phosphatidylethanolamine liposomes enhances the in vitro cytotoxicity and in vivo intratumor drug accumulation against melanomas. $J$ Dermatol Sci. 2007;46(1):11-20.

30. Khan A, Khan AA, Dwivedi V, Ahmad MG, Hakeem S, Owais M. Tuftsin augments antitumor efficacy of liposomized etoposide against fibrosarcoma in Swiss albino mice. Mol Med. 2007;13(5-6): 266-276.

31. Chow LM, Baker SJ. PTEN function in normal and neoplastic growth. Cancer Lett. 2006;241(2):184-196.

32. Mayo LD, Donner DB. The PTEN, Mdm2, p53 tumor suppressoroncoprotein network. Trends Biochem Sci. 2002;27(9):462-467.

33. Rahmani A, Alzohairy M, Babiker AY, Rizvi MA, Elkarimahmad HG. Clinicopathological significance of PTEN and bcl2 expressions in oral squamous cell carcinoma. Int J Clin Exp Pathol. 2012;5(9): 965-971.
International Journal of Nanomedicine

\section{Publish your work in this journal}

The International Journal of Nanomedicine is an international, peerreviewed journal focusing on the application of nanotechnology in diagnostics, therapeutics, and drug delivery systems throughout the biomedical field. This journal is indexed on PubMed Central, MedLine, CAS, SciSearch $®$, Current Contents $\AA /$ Clinical Medicine,

\section{Dovepress}

Journal Citation Reports/Science Edition, EMBase, Scopus and the Elsevier Bibliographic databases. The manuscript management system is completely online and includes a very quick and fair peer-review system, which is all easy to use. Visit http://www.dovepress.com/ testimonials.php to read real quotes from published authors.

Submit your manuscript here: http://www.dovepress.com/international-journal-of-nanomedicine-journal 